\title{
SOME ABSOLUTELY EFFECTIVE PRODUCT METHODS
}

\author{
H.P. DIKSHIT \\ Dept of Math \& Computer Science \\ Rani Durgavati University, \\ Jabalpur 482001 India \\ J.A. FRIDY \\ Dept. of Math \& Computer Science \\ Kent State University \\ Kent, Ohio, 44242 U.S.A. \\ (Received June 20, 1991 and in revised form March 24, 1992)
}

IBSTRACT. It is proved that the product method $A(C, 1)$, where $(C, 1)$ is the Cesàro arithmetic mean matrix. is totally effective under certain conditions concerning the matrix $A$. This general result is applied to study absolute Nörlund summability of Fourier series and other related serics.

KEY WORDS AND PIIR ASES. Absolute summability, Nörlund summability.

.MMS SUBJJECT C'LASSIFICATION NUMBER. 40F05

1. IN'TRODUC:TION AND SOME RESULTS.

Let $A=\left(\alpha_{n k}\right)$ be an infinite matrix with $\alpha_{n k}$ as real numbers. $\Lambda$ given series $u=\sum_{k=0}^{\infty} u_{k}$ or the scequence of its partial sums $\left\{U_{k}\right\}$ is said to be $|A|$ summable if

$$
v_{n}=\sum_{i=0}^{\infty} \alpha_{n k} u_{k}
$$

is defined for all $n$. and $v=\sum_{n=0}^{\infty} n_{n}$ converges absolutely. $v$ is the series-to-series, A-transformation of $u$ and we denote it by $A u$. The matrix $A$ is called absolutely conservative in case the absolute convergence of $u$ implies that of $A$ A. IVe will be mainly concerned with the case in which $A$ is absolutely conservative and

$$
\sum_{k=0}^{\infty} \frac{\left|\alpha_{n k}\right|}{k+1}<\infty
$$

for cvery fixed $n \geq 0$. It may be worthwhile to mention that the condition:

$$
\sum_{k=0}^{\infty}\left|\alpha_{n k}\right|<\infty
$$

for cvery fixed $n \geq 0$. which is stronger than (1.2), is an essential requirement for the definition of . 1 u for erery $u$ for which the sequence $\left\{u_{n}\right\}$ is convergent (see Szász [10]. Lemmas 1 and 2 of ('hapter 11I).

Let $A, B$ be given matrices for which $B u$ and the $A$-transformation of $B u$, viz., $A B u$ are defined. Then the $|A|$ summability of $B u$ or equivalently the absolute convergence of $A B u$ defines the $|A B|$ summability of $u$. It may be noted that the $|A B|$ summability is, in general, different 
than the absolute summability defined by the product matrix $A . B$ (if it exists).

It may observed that the condition $\left(1.2^{\prime}\right)$ and a fortiori $(1.2)$ are automatically satisfied whenever $A$ is a lower triangular matrix (that is, $\alpha_{n k}=0$ for $k>n$ ).

Let $p=\left\{p_{n}\right\}$ be given sequence of real numbers such that $P_{n}=\sum_{k=0}^{n} p_{k} \neq 0$ and $P_{-1}=0$. $(N, p)$ matrix is the lower triangular matrix $A$ with

$$
\begin{aligned}
\alpha_{00}=1, \alpha_{n o}=0, n \geq 1, \alpha_{n k} & =\frac{P_{n-k}}{P_{n}}-\frac{P_{n-k-1}}{P_{n-1}} \\
& =\frac{P_{n} P_{n-k}-P_{n-k} P_{n}}{P_{n} P_{n-1}}, 1 \leq k \leq n,
\end{aligned}
$$

and then (1.1) defines the series-to-series $(N, p)$-transformation. The $(N, p)$-transformation with $p_{n}=1$ for $n=0,1,2, \ldots$, defines the series-to-series $(C, 1)$-transformation. given by $b_{o}=a_{o}$ and

$$
b_{n}=\frac{1}{n(n+1)} \sum_{k=1}^{n} k a_{k}=S_{n}^{1}-S_{n-1}^{1}, n \geq 1
$$

where $S_{n}^{1}=\sum_{k=0}^{n} s_{k} /(n+1)$ and $\left\{s_{n}\right\}$ is the sequence of partial sums.

For any sequence $\left\{\beta_{n}\right\}$, we write $\Delta \beta_{n}=\beta_{n}-\beta_{n+1} .\left\{\beta_{n}\right\} \in B V$ means that $\sum_{n=1}^{\infty}\left|\Delta \beta_{n}\right|<\infty$.

Let $f(t)$ be an $(L)$-integrable periodic function with period $2 \pi$. We assume, without loss of generality that the constant term of the Fourier series $F(t)$ of $f(t)$ is zero. Let $F(t)=\sum_{n=1}^{\infty} A_{n}(t)$ and $P(t)$ denotes its conjugate series: $\sum_{n=1}^{\infty} B_{n}(t)$. Also, $F^{*}(t)=\left\{n B_{n}(t)\right\}$, and $F^{\prime \prime}(t)$ is the derived series of $F(t)$. For a given point $x$, we write

$$
\begin{gathered}
\phi(t)=\phi_{o}(t)=\frac{1}{2}\{f(x+t)+f(x-t)\}, \psi(t)=\frac{1}{2}\{f(x+t)-f(x-l)\}, \\
t \phi_{1}(t)=\Phi_{1}(t)=\int_{0}^{t} \phi(u) d u,
\end{gathered}
$$

and taking $r=0$ or 1 , we say that $A$ is $\left|F_{r}\right|$-effective provided that $\phi_{r}(t) \epsilon B V(0, \pi)$ implies that $f(x)$ is $|A|$ summable. We say that $A$ is (i) $\left|\dot{F}_{1}\right|$-effective if $\int_{0}^{\pi}|\psi(t)| t^{-1} d t<\infty$ inplies that $F^{\prime}(x)$ is $|A|$ summable. (ii) $\left|F_{i}^{*}\right|$-effective if $\psi(t) \epsilon B V(0, \pi)$ implies that $F^{*}(x)$ is $|A|$ summable. (iii) $\left|F_{1}^{\prime}\right|$-effective if $\left\{1^{-1} \ell^{\prime}(t)\right\} \in B V^{\prime}(0, \pi)$ inplies that $F^{\prime}(x)$ is $|\lambda|$ summable and absolutely total effective if it is effective in each of the senses defined above.

Unless stated otherwise, $0=[\pi / t]$, i.e., the greatest integer not greater than $\pi / t$, and $K^{\circ}$ denotes a positive constant not necessarily the same at each occurrence.

For a general matrix $A$. Tripathy [11] (see also Kuttner and Tripathy [s]) and, Kuttner and Sahney [i] have obtained sufficient conditions so that $A$ is $\left|F_{0}\right|$-effective. The restrictions imposed on $A$ in [II] are quite general. but it is usually difficult to verify them for special case: of interest. The nature of the corresponding conditions used in [7] is such that they can be easily verified, and, therefore the following result due to liuttner and Sahney has the advantage of having some direct applications.

THEOREM A. Let the matrix $A$ be absolutely conservative and $\alpha_{n k} \geq 0$ for all $n, k$. Suppose that either

(a) for each fixed $n$, there is a positive integer $r(n)$ such that $\left\{\alpha_{n k}\right\}$ is nondecreasing for $1 \leq$ $k \leq r(n)$ and nonincreasing for $k \geq r(n)$, or 
(b) for each fixed $n$, there is a posttive integer $s(n)$ such that $\left\{\alpha_{n k} / k\right\}$ is nondecreasing for $1 \leq k \leq s(n)$ and nonincreasing for $k \geq s(n)$.

Suppose also that in case (a). for $k_{0} \geq 1$

$$
\sum_{r(n) \geq 2 k_{0}} \frac{1}{r(n)} \sum_{k=r(n)-k_{0}}^{r(n)+k_{0}} \alpha_{n k}=O(1)
$$

and in case (b), for $k_{o} \geq 1$

$$
\sum_{s(n) \geq 2 k_{0}} \frac{1}{s(n)} \sum_{k=s(n)-k_{0}}^{s(n)+k_{0}} \alpha_{n k}=O(1)
$$

Then $A$ is $\left|F_{0}\right|$-effective.

Starting with an absolutely conservative method $A$. we obtain in the present paper a suflicient condition that connects the proof of absolutely total effectiveness of $A(C, 1)$ with the proof of $\left|F_{v}\right|$-effectiveness of the $A$ matrix. As we shall see. such a result has some interesting applications. We will first prove the following.

THEOREM 1. If $A$ is absolutely conservative, (1.2) holds and

$$
\sum_{n=1}^{\infty}\left|\sum_{k=20}^{\infty} \alpha_{n k} \frac{\exp (i k \cdot t)}{k}\right|=O(1)
$$

for lc $(0, \pi]$. then $A(C, 1)$ is absolutcly total cffective.

We shall obtain the following corollaries to Theorem 1.

COROLLARY 1. If $A$ satisfies the hypotheses of Theorem $A$ and $(1.2)$, then $A(C, 1)$ is ،bsolutely total effective.

COROLLARY 2. If $\left\{p_{n}\right\}$ is any sequence such that $\left\{(n+1) p_{n} / P_{n}\right\}=\left\{R_{n}\right\} \in B V$ and

$$
\left\{\frac{1}{P_{n}} \sum_{k=0}^{n}(k+1)^{-1}\left|P_{k}\right|\right\}=\left\{S_{n}^{*}\right\}, B \text {. }
$$

i.e. $\left\{S_{n}^{*}\right\}$ is a bounded sequence, then $(N, p)(C, 1)$ is absolutely total effective.

As we shall sce in the last section of the present paper, a special case of Corollary 1 provides a result which inclucles, inter alia, sharper results than those proved clsewhere. (CF. [3], Theorems 1 and 2 ). On the other hand. Corollary 2 includes inter alia the results contained in [4] ('Theorems 1 and 2$)$.

In Section 4, we obtain another theorem which provides a somewhat more direct sufficient condition for $\left|F_{1}\right|$ - and $\left|F_{1}^{\prime}\right|$-effectiveness of $A(C, 1)$. This theorem. which is less general than Theorem 1 in the sense that it does not provide the absolutely total effectiveness of $A(C, 1)$, is readily applicable. and we deduce from it considerably shorter proofs of some earlier results (see [1]. Theorems 1 and 2).

\section{SOME PRELIMINARY RESULTS.}

We shall need the following lemmas for the proof of Theorem 1 and its corollaries.

LEMMA 1. The necessary and sufficient condition that $A$ be absolutely conservative is that for all $k \geq 0$,

$$
\sum_{n=0}^{\infty}\left|\alpha_{n k}\right|=O(1)
$$

The result of Lemma 1 is well known. See, e.g., [9] or [6]. 
LEMMA 2. Let $\left\{a_{n}\right\}$ be a given sequence; then for any number $x$ we have

$$
-(1-x) \sum_{k=m}^{n} a_{k} x^{k}=\sum_{k=m}^{n} \Delta a_{k} x^{k+1}-a_{m} x^{m}+a_{n+1} x^{n+1},
$$

where $m$ and $n$ are integers such that $n \geq m \geq 0$.

The proof of Lemma 2 is a straight forward calculation.

LEMNA 3. If $A$ satisfies the hypotheses of Theorem A, then (1.7) holds.

PROOF. Observing that $(\sin k t) / k$ is the imaginary part of $\exp (i k t) / k$, we shall prove (1.7) by following closely the proof of Theorem $A$, which contains the proof for $\sum_{n}\left|b_{n}(t)\right|=O(1)$ with $\sum_{n} b_{n}(t)$ as the $A$-transformation of $\sum_{k=1}^{\infty}(\sin k t) / k$. Thus, considering those values of $n$ (if any) for which $r(n) \leq 20$ (or $s(n) \leq 20)$, we have that $\left\{\alpha_{n k} / k\right\}$ is nonnegative and nonincreasing for $k \geq 20$. Therefore, by an application of Abel's Lemma to the inner sum and using the fact that the partial sums $\sum \exp (i k t)$ are $O(1 / t)$, we have

$$
\sum_{\substack{(n)<20 \\(\operatorname{or} s(n)<20)}}\left|\sum_{k=2 \theta}^{\infty} \alpha_{n k} \frac{\exp (i k \cdot t)}{k}\right|=O\left\{\sum_{n=1}^{\infty} \alpha_{n, 2 \theta}\right\}=O(1) ;
$$

the $O(1)$ estimate is obtained from Lemma 1 and the hypothesis that $A$ is absolutely conservative. Considering the case (b), we follow exactly the argument in [7], p. 413 , to get

$$
\sum_{s(n) \geq 2 \theta}\left|\sum_{k=2 \theta}^{\infty} \alpha_{n k} \frac{\exp (i k t)}{k}\right|=O(1) .
$$

In case (a), the part of the sum for $k \geq r(n)-0$ may be dealt with as in [7], p. 413, and it remains to show that when $r(n)-0>20$, then

$$
\sum_{r(n)>3 \theta}\left|\sum_{k=2 \theta}^{r(n)-\theta} \alpha_{n k} \frac{\exp (i k t)}{k}\right|=O(1)
$$

To verify this we apply Lemma 2 and get (cf. [7],p. 113)

$$
\begin{gathered}
\left|\sum_{k=2 \theta}^{r(n)-\theta} \frac{\alpha_{n k}}{k} \exp (i k \cdot t)\right| \leq \\
K t^{-1}\left[\sum_{k=2 \theta}^{r(n)-\theta}\left|\Delta_{k}\left(\alpha_{n k} / k\right)\right|+\frac{\alpha_{n, 2 \theta}}{\theta}+\frac{\alpha(n, r(n)-\theta+1)}{r(n)-\theta+1}\right],
\end{gathered}
$$

where, for convenience. we write $\alpha(n, k)$ for $\alpha_{n k}$. The rest of the proof of (2.2) follows directly from [i]. This completes the proof of the present lemma.

LEMMA 1. If $\left\{p_{n}\right\}$ satisfies the hypotheses of Corollary 2 , then $(N, p)$ is absolutely regular and a forthor absolutcly conservative.

Lemma 4 may be proved by following the proof of Lemma 10 in [4].

LEMMA 5. If $\left\{R_{n}\right\} \epsilon B$, then $\left\{S_{n}^{*}\right\} \in B$ implies that $P_{n}^{*}=\sum_{k=0}^{n}\left|p_{k}\right|=O\left(\left|P_{n}\right|\right)$ and $\left\{S_{n}^{*}\right\} \epsilon B$ is equivalent to the following:

$$
\left|P_{n}\right| \sum_{k=n}^{\infty} \frac{1}{k\left|P_{k}\right|}=O(1), n=1,2, \ldots
$$

The first part of the lemma follows directly when we observe that

$$
\sum_{k=0}^{n}\left|p_{k}\right|=\sum_{k=0}^{n} \frac{\left|R_{k} P_{k}\right|}{k+1}
$$

and appeal to the hypotheses $\left\{R_{n}\right\} \in B$ and $\left\{S_{n}^{*}\right\} \in B$. The second part follows as a special case of 
an earlier result (see [5], Lemma 3; see also [2]).

LEMMA 6. If $P_{n}^{*}=O\left(\left|P_{n}\right|\right)$, then uniformly for all $r, s(r \geq s \geq 0)$

$$
\left|\sum_{k=0}^{r} P_{k} \exp (-i k t)\right| \leq k t^{-1}\left|P_{r}\right|
$$

uniformly in $0<t \leq \pi$.

Lemma 6 may be proved by applying Abel's transformation and using the result that the partial sums $\sum \exp (-i k t)$ are $O(1 / t)$.

LEMMA 7. If $\left\{p_{n}\right\}$ satisfics the hypotheses of Corollary 2 , then $(1.7)$ holds for the $(N, p)$ transformation defined by (1.3).

PROOF. Consider the condition:

$$
N=\sum_{n=\theta}^{\infty}\left|\sum_{k=\theta}^{n}\left[\frac{P_{n-k}}{P_{n}}-\frac{P_{n-k-1}}{P_{n-1}}\right] \frac{\exp (i k t)}{k}\right|=O(1),
$$

which is obtained from (1.7) by replacing 20 by 0 .

We shall first prove (2.3). Now observing that

$$
\frac{P_{k}}{P_{n}}-\frac{P_{k-1}}{P_{n-1}}=\frac{1}{(n+1) P_{n-1}}\left[P_{k}\left(R_{k}-R_{n}\right)+(n-k) p_{k}\right]
$$

we have

$$
\begin{gathered}
N \leq \sum_{n=0}^{\infty} \frac{1}{n\left|P_{n-1}\right|}\left|\sum_{k=0}^{n-\theta} P_{k} \frac{\exp (-i k t)}{n-k} \sum_{r=k}^{n-1}\left(\Delta R_{r}\right)\right| \\
+\sum_{n=0}^{\infty} \frac{1}{n\left|P_{n-1}\right|}\left|\sum_{k=0}^{n-\theta} p_{k} \exp (-i k t)\right| \\
=N_{1}+N_{2},
\end{gathered}
$$

say. Applying $\Lambda$ bel's Lemma to the inner sum, we have

$$
N_{1} \leq \kappa t \sum_{n=\theta}^{\infty} \frac{1}{n\left|P_{n-1}\right|} \max _{0 \leq m \leq n-\theta}\left|\sum_{k=m}^{n-\theta} P_{k} \exp (-i k t) \sum_{r=k}^{n-1} \Delta R_{r}\right| .
$$

Writing $r^{\prime}$ for $\min (r, n-0)$, we see that by a change in the order of summation. we have for any $0 \leq m<n$

by virtue of Lemma 6 . Thus,

$$
\begin{gathered}
\left|\sum_{k=m}^{n-\theta} P_{k} \exp p(-i k \cdot t) \sum_{r=k}^{n-1} \Delta R_{r}\right|=\left|\sum_{r=m}^{n-1} \Delta R_{r} \sum_{k=m}^{r^{\prime}} P_{k} \exp (-i k \cdot t)\right| \\
\leq K_{1} t^{-1} \sum_{r=0}^{n-1}\left|\Delta R_{r}\right|\left|P_{r}\right|
\end{gathered}
$$

$$
\begin{aligned}
N_{1} & \leq K_{1} \sum_{n=1}^{\infty} \frac{1}{n\left|P_{n-1}\right|} \sum_{r=0}^{n-1}\left|\Delta R_{r}\right|\left|P_{r}\right| \\
& =K_{1} \sum_{r=0}^{\infty}\left|\Delta R_{r}\right|\left|P_{r}\right| \sum_{n=r}^{\infty} \frac{1}{(n+1)\left|P_{n}\right|}=O(1),
\end{aligned}
$$

by virtue of Lemma 5 and the hypothesis that $\left\{R_{n}\right\} \epsilon B V$.

Taking $\sum_{a}^{b}=0$ if $a>b$, breaking the range for $k$ into tivo parts viz.. $k<\theta$ and $k \geq \theta$ and observing that by Lemma $5, P_{n}^{*}=O\left(\left|P_{n}\right|\right)$, we have

$$
N_{2} \leq K_{1}\left|P_{\theta}\right| \sum_{n=\theta}^{\infty} \frac{1}{n\left|P_{n-1}\right|}+K_{1} \sum_{n=\theta}^{\infty} \frac{1}{n\left|P_{n-1}\right|}\left|\sum_{k=\theta}^{n-\theta} p_{k} \exp (-i k t)\right| .
$$

Since $p_{k}=P_{k}\left\{R_{k} /(k+1)\right\}$ and the partial sums of $\sum \exp (-i k t)=O(1 / t)$, an application of the 
Abel's transformation shows that for any $m$ with $\theta \leq m \leq n$, we have

$$
\begin{aligned}
\left|\sum_{k=m}^{n} p_{k} \exp (-i k t)\right| & \leq K t^{-1} \sum_{k=m}^{n-1}\left|\Delta\left(\frac{R_{k} P_{k}}{k+1}\right)\right|+K t^{-1} \frac{\left|P_{n}\right|}{n} \\
& \leq K \sum_{k=\theta}^{n}\left\{\left|\Delta R_{k}\right|+\frac{t^{-1}}{k(k+1)}\right\}\left|P_{k}\right|+K t^{-1} \frac{\left|P_{n}\right|}{n} .
\end{aligned}
$$

'Thus. using the result of Lemma 5 and a suitable change in the order of summations, we have

$$
N_{2} \leq K+K \sum_{k=\theta}^{\infty}\left\{\left|\Delta R_{k}\right|+\frac{t^{-1}}{k(k+1)}\right\}\left|P_{k}\right| \sum_{n=k}^{\infty} \frac{1}{n\left|P_{n-1}\right|}=O(1),
$$

because $\left\{R_{n}\right\} \in B V$.

Combining (2.5) with (2.6), we prove (2.3). Since the above proof remains valid if 0 is replaced by 20 , we deduce (1.7) from the proof of (2.3).

3. PROOF OF 'THEOREM 1 AND ITS COOROLLARIES.

(I). $\left|F_{1}\right|$-effectiveness: We have assumed without loss of generality that the constant term in $f(x)$ is zero so that

$$
\Phi_{1}(\pi)=\int_{0}^{\pi} \phi(t) d t=0
$$

Now. we have

$$
A_{n}(x)=\frac{2}{\pi} \int_{0}^{\pi} \phi(t) \cos n t d t .
$$

Denoting by $\sum A_{k}^{1}(x)$ the series-to-serics $(C, 1)$-transformation of $F(x)$, we have

$$
A_{k}^{1}(x)=\frac{2}{\pi} \int_{0}^{\pi} \phi(t) \frac{1}{k(k+1)} \sum_{r=1}^{k} r \cos r t d t .
$$

Integrating by parts we see that $A_{k}^{1}(x)$ is the real part of

$$
\begin{aligned}
\frac{2}{\pi} \int_{0}^{\pi} \omega(t) \delta_{k}(t) d t & =\frac{2}{\pi} \int_{0}^{\pi} t \varphi_{1}(t) \phi \frac{d}{d t} \delta_{k}(t) d t \\
& =\frac{2}{\pi} \int_{0}^{\pi}\left\{t \delta_{k}(t)-\int_{0}^{t} \delta_{l}(u) d u\right\} d \phi_{1}(l)
\end{aligned}
$$

where

$$
\delta_{k}(t)=\frac{1}{k \cdot(k+1)} \sum_{r=1}^{k} r \exp (i r t) .
$$

Thus, if $\sum b_{n}(x)$ denotes the $A(C, 1)$-transformation of $F(x)$, then we have

$$
\begin{aligned}
b_{n}(x) & =-\frac{2}{\pi} \sum_{k=1}^{\infty} \alpha_{n k} \int_{0}^{\pi} t\left[\operatorname{Re} \delta_{k}(t)\right] d \phi_{1}(t) \\
& +\frac{2}{\pi} \sum_{k=1}^{\infty} \alpha_{n k} \int_{0}^{\pi}\left(\int_{0}^{t}\left[\operatorname{Re} \delta_{k}(u)\right] d u\right) d \phi_{1}(t) .
\end{aligned}
$$

Now it is easily seen that $\delta_{k}(t)=O(1 / k t)$, therefore,

$$
t \sum_{k=1}^{\infty}\left|\alpha_{n k}\right|\left|\delta_{k}(t)\right|=O(1)
$$

by virtue of the condition (1.2). Next we notice that

$$
\int_{0}^{t}\left[\operatorname{Re} \delta_{k}(u)\right] d u=\frac{1}{k(k+1)} \sum_{r=1}^{k} \sin r t
$$

Thus,

$$
\sum_{k=1}^{\infty}\left|\alpha_{n k}\right|\left|\int_{0}^{t}\left[\operatorname{Re} \delta_{k}(u)\right] d u\right|=O(1)
$$


by virtuc of the condition (1.2). In view of (3.4) and (3.5), we have

$$
b_{n}(x)=-\frac{2}{\pi} \int_{0}^{\pi}\left[t R(n, t)-\sum_{k=1}^{\infty} \alpha_{n k} \int_{0}^{t} \operatorname{Re} \delta_{k}(u) d u\right] d \phi_{1}(t)
$$

where $R(n, t)$ is the real part of

$$
J(n, t)=\sum_{k=1}^{\infty} \alpha_{n k} \delta_{k}(t)
$$

Since by hypothesis $\int_{o}^{\pi}\left|d \phi_{1}(t)\right| \leq \kappa$, in order to prove that $A(C, 1)$ is $\left|F_{1}\right|$-effective, it is sufficient to show that the following estimates hold for $t \epsilon(0, \pi)$ :

$$
\begin{gathered}
S_{1}=t \sum_{n=1}^{\infty}|J(n, t)|=O(1) \\
S_{2}=\sum_{n=1}^{\infty}\left|\sum_{k=1}^{\infty} \alpha_{n k} \int_{0}^{t} \operatorname{Re} \delta_{k}(u) d u\right|=O(1) .
\end{gathered}
$$

We first proceed to prove (3.7). Breaking the range of summation for the inner sum into $1 \leq k<20$ and $20 \leq k$, we use for the former range the fact that $\delta_{k}(t)=O(1)$, while for the latter range we replace $\delta_{k}(t)$ by the following expression which is equal to it:

$$
\frac{(1-\exp (i t))^{-1}}{k+1}\left\{\frac{1}{k} \sum_{r=1}^{k} \exp (i r t)-\exp (i[k+1] t)\right\}
$$

Thus, we write

$$
\begin{aligned}
S_{1} \leq K t \sum_{n=1}^{\infty} & \sum_{k=1}^{20-1}\left|\alpha_{n k}\right|+K \sum_{n=1}^{\infty}\left|\sum_{k=2 \theta}^{\infty} \frac{\alpha_{n k}}{k}\left(1-\frac{1}{k+1}\right) \exp (i[k+1] t)\right| \\
& +K \sum_{n=1}^{\infty}\left|\sum_{k=2 \theta}^{\infty} \alpha_{n k} \frac{1}{k(k+1)} \sum_{r=1}^{k} \exp (i r t)\right| \\
& \leq K t \sum_{k=1}^{2 \theta} \sum_{n=1}^{\infty}\left|\alpha_{n k}\right|+K \sum_{n=1}^{\infty}\left|\sum_{k=2 \theta}^{\infty} \alpha_{n k} \frac{\exp (i k t)}{k}\right| \\
& +K \theta \sum_{n=1}^{\infty} \sum_{k=2 \theta}^{\infty} \frac{1}{k(k+1)}\left|\alpha_{n k}\right| \\
& \leq K t \sum_{k=1}^{2 \theta} 1+K \theta \sum_{k=2 \theta}^{\infty} \frac{1}{k(k+1)} \sum_{n=1}^{\infty}\left|\alpha_{n k}\right|+O(1) \\
& =O(1)
\end{aligned}
$$

by virtue of the hypothesis (1.7) and that $A$ is absolutely conservative so that (2.1) holds. We thus prove (3.7). Next, we have

$$
\begin{aligned}
S_{2} & \leq \sum_{n=1}^{\infty}\left|\left(\sum_{k=1}^{\theta-1}+\sum_{k=\theta}^{\infty}\right) \alpha_{n k} \frac{1}{k(k+1)} \sum_{r=1}^{k} \sin r t\right| \\
& \leq t \sum_{k=1}^{\theta-1} \sum_{n=1}^{\infty}\left|\alpha_{n k}\right|+K t^{-1} \sum_{n=1}^{\infty} \sum_{k=\theta}^{\infty} \frac{1}{k(k+1)}\left|\alpha_{n k}\right| \\
& =O(1),
\end{aligned}
$$

by reasoning parallel to that used in the preceding paragraph. Thus (3.8), holds and we complete the proof of $\left|F_{1}\right|$-effectiveness of $A(C, 1)$.

(II). $\left|\bar{F}_{1}\right|$-effectiveness: Since, for the serics $\bar{F}(x)$, 


$$
B_{n}(x)=\frac{2}{\pi} \int_{0}^{\pi} \psi(t) \sin n t d t
$$

it follows from (3.4) that

$$
\bar{b}_{n}(x)=\frac{2}{\pi} \int_{0}^{\pi} \psi(t) I(n, t) d t
$$

where $I(n, l)$ is the imaginary part of $J(n, t)$ and $\sum_{n} \bar{b}_{n}(x)$ is the $A(C, 1)$-transformation of $\bar{F}(x)$. But by hypothesis $\int_{0}^{\pi} t^{-1}\left|b^{\prime}(t)\right| d t \leq \Lambda^{;}$; therefor, in order to prove the $\left|\bar{F}_{1}\right|$-effectiveness of $A(C, 1)$ it is sufficient to show that

$$
t \sum_{n=1}^{\infty}|I(n, t)|=O(1) .
$$

This follows directly from (3.7), and we complete the proof of the $\left|\bar{F}_{1}\right|$-effectiveness of $\therefore\left(c^{\prime} .1\right)$.

(III). $\left|F_{i}^{*}\right|$-effectiveness: For the sequence $F^{*}(x)$,

$$
n B_{n}(x)=\frac{2}{\pi} \int_{0}^{\pi} \cos n t d \psi^{\prime}(t)
$$

since $\psi(\pi)=0$. Thus. the terms of the corresponding series are obtained as

$$
u_{n}(x)=\frac{4}{\pi} \int_{0}^{\pi} \sin \left(\frac{1}{2} t\right) \sin \left(n-\frac{1}{2}\right) t d \psi(t) .
$$

Denoting by $\sum u_{n}^{1}(x)$ the series-to-series $(C, 1)$-transformation of $\sum u_{n}(x)$, we have

$$
u_{n}^{1}(x)=\frac{1}{\pi} \int_{0}^{\pi} \sin \left(\frac{t}{2}\right)\left\{\cos \left(\frac{t}{2}\right) \operatorname{Im} \delta_{k}(t)-\sin \left(\frac{t}{2}\right) R c \delta_{k}(t)\right\} d \psi(t) .
$$

Thus, if $\sum b_{n}^{*}(x)$ denotes the $A(C, 1)$-transformation of $F^{*}(x)$ then in view of (3.4), we have

$$
b_{n}^{*}(x)=\frac{4}{\pi} \int_{0}^{\pi} \sin \left(\frac{1}{2} t\right)\left\{\cos \left(\frac{1}{2} t\right) I(n, t)-\sin \left(\frac{1}{2} t\right) R(n, t)\right\} d \psi(t) .
$$

But by hypothesis $\psi(t) \epsilon B V(0, \pi)$; therefore, in order to prove the $\left|F_{1}^{*}\right|$-effectiveness of $A(C, 1)$ it is sufficient to show that (3.7) holds. We thus complete the proof of the $\left|F_{1}^{*}\right|$-effectiveness of $A(C, 1)$.

(IV). $\left|F_{1}^{\prime}\right|$-effectiveness: For the series $F^{\prime}(x)=\sum u_{n}(x)$, we have

$$
u_{n}(x)=-\frac{2}{\pi} \int_{0}^{\pi} \psi(t) \frac{d}{d t}(\cos n t) d t,
$$

and. therefore,

$$
u_{n}^{1}(x)=-\frac{2}{\pi} \int_{0}^{\pi} \frac{\psi(t)}{t} t \operatorname{Re}\left\{\frac{d}{d t} \delta_{k}(t)\right\} d t .
$$

Following the proof of the $\left|F_{1}\right|$-effectiveness part, we deduce $\left|F_{1}^{\prime}\right|$-effectiveness of $A(C, 1)$ from (3.7) and (3.8) by comparing (3.9) with (3.2) and then appealing to the hypothesis that $(\psi(t) / t) \epsilon B V(0, \pi)$.

Since $\phi(t) \epsilon B V(0, \pi)$ implies that $\phi_{1}(t) \epsilon B V(0, \pi)$, the $\left|F_{0}\right|$-effectiveness of $A(C, 1)$ is included in its $\left|F_{1}\right|$-effectiveness. Thus combining (I) - (IV), we complete the proof of Theorem 1.

To prove Corollary 1 we note that if $A$ satisfies the hypotheses of Theorem A, then Lemma 3 ensures the condition (1.7) of Theorem 1. This together with (1.2) implies the conclusion of Theorem 1 , which is also the conclusion of Corollary 1 . Since for the $(N, p)$ transformation, (1.2) holds automatically we use Lemma 4 and Lemma 7 to see that the hypotheses of Theorem 1 are satisfied and in conclusion we obtain Corollary 2.

\section{ADDITIONAL RESULTS.}

We shall also prove the following. 
'TIIEOREM 2. If $A$ is absolutcly conservative and the condition $g(t) \subset B V(0, \pi)$ implies the $|A|$ summability of $\sum w_{n}$, where

$$
w_{n}=\int_{0}^{\pi} g(t) \cos \left(n+\frac{1}{2}\right) t d t
$$

then the $A(C, 1)$ is $\left|F_{1}\right|$ - and $\left|F_{1}^{\prime}\right|$-effective.

Comparing the hypotheses of Theorem 1 with those of Theorem 2, we observe that the condition (1.T) of Theorem 1 is directly associated with the $|A|$ summability of the series $\sum_{k=1}^{\infty} \exp (i k t) / k$. Similarly, the corresponding condition of Theorem 2 is closely associated with $\left|F_{0}\right|$-effectiveness of $A$, which is usually proved by establishing the stronger result that $\sum\left|c_{n}(t)\right|=O(1)$ where $\sum c_{n}(t)$ is the $A$-transformation of $\sum(\sin k t) / k$.

We shall need the following additional lemmas for the proof of Theorem 2.

LEMM $\lambda$ s. For any fixed $\lambda \geq 0$, we set

$$
u(t)=\sum_{k=1}^{\infty}\{\sin (k+\lambda) t\} /(k+\lambda) .
$$

If $\sum_{n} c_{n}(l)$ is either (i) the A-transformation of $u(t)$ such that $A$ satisfies the hypotheses of 'Theorem $A$. or (ii) the $(N, p)$-transformation of $u(t)$ such that $\left\{p_{n}\right\}$ satisfies the hypotheses of ('orollary 2. then $\sum_{n}\left|c_{n}(t)\right|=O(1)$ for $t c[0, \pi]$.

PROOF. In view of Lemma 4 and the hypotheses of Theorem $A$ we notice that both $A$ and $(N, p)$ are absolutely conservative and it follows that in order to prove Lemma 8 it is enough to prove it with $u(t)$ replaced by $v(t)=\sum_{k}\{\sin (k+\lambda) t\} / k$. But we may prove this by following closely the proof of Lemma 8 for the case $\lambda=0$ which is contained in [7], [2] (proof of Theorem C). This completes the proof of the lemma.

LEMMA 9. If $g(t) \epsilon B V(0, \pi)$, then $\left\{V_{n}\right\} \epsilon B V$, where

$$
\pi V_{n}=\frac{1}{n+1} \int_{0}^{\pi} g(t)\left[\frac{\sin \frac{1}{2}(n+1) t}{\sin \frac{1}{2} t}\right]^{2} d t
$$

PROOF. Let $\sum A_{n}(x)$ denote the Fourier series at $t=x$, of a $2 \pi$-periodic function $f(t)$ such that $g(t)=\frac{1}{2}\{f(x+t)+f(x-t)\}$. Then we see that $V_{\eta}=\sum_{k=0}^{n} s_{k}(x) /(n+1)$, where $\left\{s_{k}(x)\right\}$ is the sequence of partial sums of $\sum A_{k}(x)$. Thus, in view of $(1.4)$ the result of the lemma is essentially that $(C, 1)$ is $\left|F_{o}\right|$-effective. The lemma now follows from Bosanquet [1] where it has been shown that $(C, \alpha)$ is $\left|F_{0}\right|$-effective for $\alpha>0$.

\section{PROOF OF THEOREM 2.}

(I). $\left|F_{1}\right|$-effectiveness: Taking $\sum a_{n}=F(x)$ in (1.4) we have

$$
\begin{aligned}
\pi S_{n}^{1} & =\frac{1}{(n+1)} \int_{0}^{\pi} \phi(t) \sum_{r=0}^{n}\left\{\sin \left(r+\frac{1}{2}\right) t / \sin \frac{1}{2} t\right\} d t \\
& =\frac{1}{(n+1)} \int_{0}^{\pi} \phi(t)\left\{\sin \frac{1}{2}(n+1) t / \sin \frac{1}{2} t\right\}^{2} d t .
\end{aligned}
$$

Using (3.1) and integration by parts (cf. [4], p. 480), we have

$$
\begin{aligned}
S_{n}^{1}= & -\frac{1}{2 \pi} \int_{0}^{\pi} \frac{\Phi_{1}(t)}{\sin \frac{1}{2} t}\left\{\frac{\sin (n+1) t}{\sin \frac{1}{2} t}\right\} d t \\
& +\frac{1}{\pi(n+1)} \int_{0}^{\pi} \frac{\Phi_{1}(t)}{\sin \frac{1}{2} t}\left\{\cos \frac{1}{2} t\right\}\left\{\frac{\sin \frac{1}{2}(n+1) t}{\sin \frac{1}{2} t}\right\}^{2} d t
\end{aligned}
$$




$$
=W_{n}+V_{n} \text {, }
$$

say. 'Thus. in order to prove that $A(C, 1)$ is $\left|F_{1}\right|$-effective it is enough to show that both $\sum\left(V_{n}-\right.$ $\left.V_{n-1}\right)$ and $\sum\left(W_{n}-W_{n-1}\right)$ are $|A|$ surnmable. That the former of these is $|A|$ summable follows from Lemma 9 and the hypothesis that $A$ is absolutely conservative when we observe that $D_{1}(t) c B V(0, \pi)$. Next we see that

$$
W_{n}-W_{n-1}=-\frac{1}{\pi} \int_{0}^{\pi} \frac{\Phi_{1}(t)}{\sin \frac{1}{2} t} \cos \left(n+\frac{1}{2}\right) t d t
$$

and, therefore. $|A|$ summability of $\sum\left(W_{n}-W_{n-1}\right)$ follows from the hypotheses of Theorem 2 when we appeal to the fact that $\left(\Phi_{1}(t) / \sin \frac{1}{2} t\right) \epsilon B V(0, \pi)$. This completes the proof of the $\left|F_{1}\right|$-effectiveness of $A\left(C^{\prime}, 1\right)$.

(II). $\left|F_{1}^{\prime}\right|$-effectiveness : Taking $\sum a_{n}=F^{\prime}(x)$ in (1.4), we have (cf. [4], p. 480)

$$
\begin{aligned}
S_{n}^{1}= & -\frac{1}{\pi(n+1)} \int_{0}^{\pi} \psi(t) \frac{d}{d t}\left\{\frac{\sin \frac{1}{2}(n+1) t}{\sin \frac{1}{2} t}\right\}^{2} d t \\
= & -\frac{1}{2 \pi} \int_{0}^{\pi} \frac{\psi(t)}{\sin \frac{1}{2} t}\left\{\frac{\sin (n+1) t}{\sin \frac{1}{2} t}\right\} d t \\
& +\frac{1}{\pi(n+1)} \int_{0}^{\pi} \frac{\psi(t)}{\sin \frac{1}{2} t}\left(\cos \frac{1}{2} t\right)\left\{\frac{\sin \frac{1}{2}(n+1) t}{\sin \frac{1}{2} t}\right\}^{2} d t .
\end{aligned}
$$

Comparing (5.2) with (5.1) and observing that by hypothesis $\left\{\psi(t) / \sin \frac{1}{2} t\right\} \epsilon B V(0, \pi)$, we prove that $A(C, 1)$ is $\left|F_{1}^{\prime}\right|$-effective by following the preceding proof. This completes the proof of Theorem 2.

\section{REMARKS.}

We observe that the hypothesis concerning the $|A|$ summability of $\sum_{n=1}^{\infty} w_{n}$ was applied in the proof of Theorem 2 for $g(t)=\Phi_{1}(t) / \sin \frac{1}{2} t$ and $\psi(t) / \sin \frac{1}{2} t$. Thus, in view of $(3.1)$ and $\psi(\pi)=0$ we may take $g(\pi)=0$. Therefore, on integration by parts we see from (4.1) that

$$
w_{n}=-\int_{0}^{\pi}\left\{\sin \left(n+\frac{1}{2}\right) t\right\} /\left(n+\frac{1}{2}\right) d g(t) .
$$

If we now denote by $\sum y_{n}$ the $A$-transform of $\sum w_{n}$ and assume (1.2) and that $g(t) \epsilon B V(0, \pi)$, then

$$
\begin{aligned}
y_{n} & =-\sum_{k=1}^{\infty} \alpha_{n k} \int_{0}^{\pi}\left\{\sin \left(k+\frac{1}{2}\right) t\right\} /\left(k+\frac{1}{2}\right) d g(t) \\
& =-\int_{0}^{\pi} c_{n}(t) d g(t)
\end{aligned}
$$

and a sufficient condition for the $|A|$ summability of $\sum_{n=1}^{\infty} w_{n}$ is that $\sum\left|c_{n}(t)\right|=O(1)$ where $\sum c_{n}(t)$ is the $A$-transformation of $\sum_{k=1}^{\infty} \sin \left(k+\frac{1}{2}\right) t /\left(k+\frac{1}{2}\right)$. This is satisfied, however, by virtue of Lemma 8, when either $A$ satisfies the hypotheses of Corollary 1 or $A$ is $(N, p)$ and satisfies the hypotheses of Corollary 2. Thus we deduce from Theorem 2, $\left|F_{1}\right|$ - and $\left|F_{1}^{\prime}\right|$-effective parts of Corollary 1 and Corollary 2. It is interesting to observe that the foregoing arguments provide remarkably shorter proofs of some earlier results ([4], Theorems 1 and 2).

Following [7], we observe that if $\left\{p_{n}\right\}$ is nonnegative, nonincreasing and $\left\{S_{n}^{*}\right\} \in B$. then the $(N, p)$ satisfies the hypotheses of Theorem $\mathrm{A}$, and Corollary 1 provides the following result which inter alia includes sharper results than those proved elsewhere ([3], Theorems 1 and 2). 
COROLLARY 3. If $\left\{p_{n}\right\}$ is nonnegative, nonincreasing, and $\left\{S_{n}^{*}\right\} \in B$, then the $(N, p)(C, 1)$ is absolutely total effective.

Corollary 1 also shows that if, in Corollary 2, we impose the additional restriction that $\left\{p_{n}\right\}$ is nonnegative and nonincreasing, then the hypothesis $\left\{R_{n}\right\} \in B V$ may be deleted. It may be mentioned here. however, that there exist sequences $\left\{p_{n}\right\}$ which satisfy the hypotheses of ('orollary 2 but which are not even monotonic. The absolutely total effectiveness of the ('esàro Inatrix $(C, \delta)$ for $\delta>1$ was initiated by Bosanquet [1] and was completed subsequently. As a special case of Corollaries 1 and 2 , we obtain the absolutely total effectiveness of the $(C, \delta)$ matrix for $\delta>1$.

The authors should like to thank the referee for some helpful suggestions.

\section{REFERENCES}

1. BOSANQUET, L.S. The absolute Cesàro summability of a Fourier series, Proc. London Math. Soc., 41(1936), 517-528.

2. DIKSHIT, H.P. Absolute summability of some series related to a Fourier series, J. Australian Math. Soc., 13(1971), 7-14.

3. DIKSHIT, H.P. Absolute total effective $\left(N, p_{n}\right)(C, 1)$ method, Pacific J. Math., 41(1972). 89-98.

4. DIKSHIT, H.P. Absolute summability of a Fourier series and its derived series by a product method, J. Australian Math. Soc., 14(1972), 470-181.

5. DIKSHIT, H.P. and KUMAR, A. Absolute total-effective $\left(N, p_{n}\right)$ means 1, Proc. Cambridge Phil. Soc., 77(1975), 103-108.

6. FRIDY, J.A. A note on absolute summability, Proc. Amer. Math. Soc., 20(1969), 285-286.

7. KUTTNER, B. and SAHNEY, B.N. On the absolute matrix summability of Fourier series, Pacific J. Math., 43(1972), 407-419.

8. KUTTNER, B. and TRIPATHY, N. An inclusion theorem for Hausdorff summability method associated with functional integrals, Quart. J. Math(Oxford), (2),22(1971), 299-308.

9. MEARS, F.M. Absolute regularity and the Nörlund means, Annals of Math, 38(1937), 594-601.

10. SZÁSZ. O. Introduction to the Thcory of Divergent Series, New York, 1952.

11. TRIPATHY, N. On the absolute Hausdorff summability of Fourier series, J. London Math. Soc., $14(1969), 15-25$. 


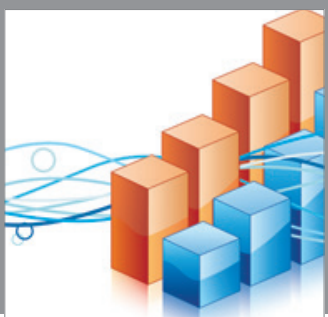

Advances in

Operations Research

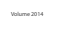

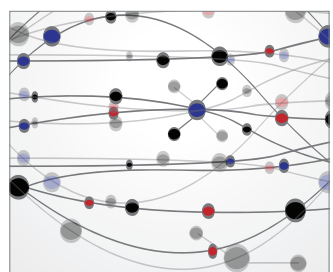

\section{The Scientific} World Journal
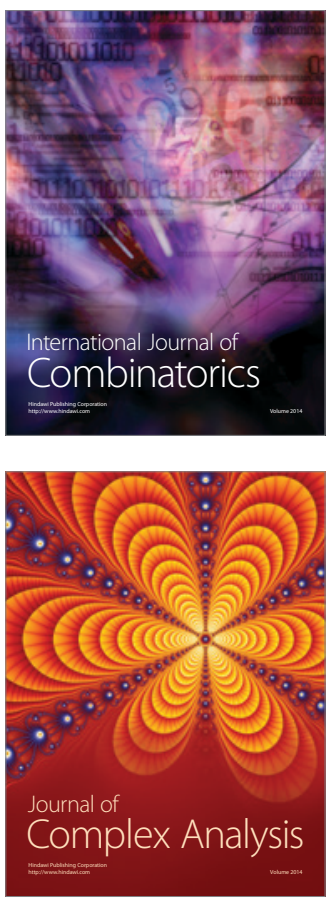

International Journal of

Mathematics and

Mathematical

Sciences
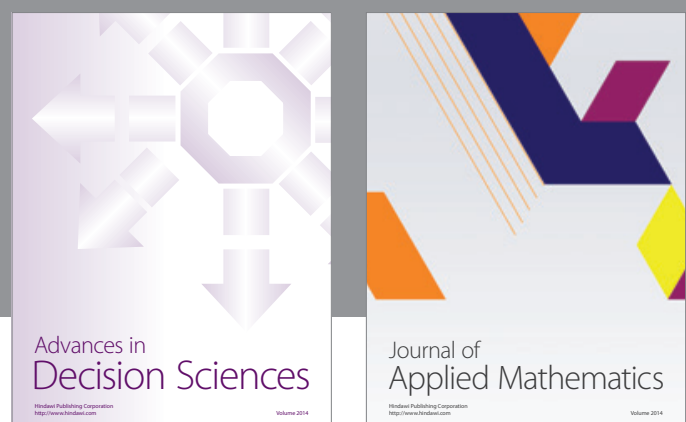

Journal of

Applied Mathematics
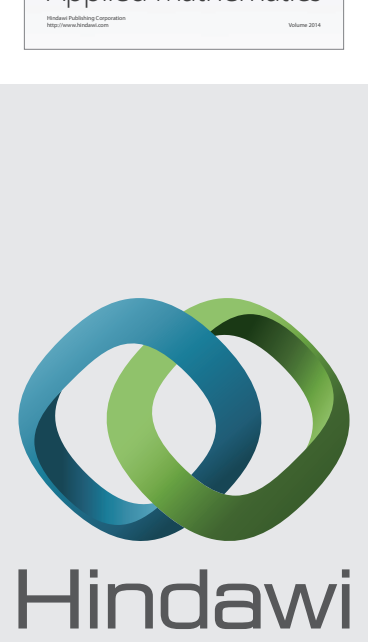

Submit your manuscripts at http://www.hindawi.com
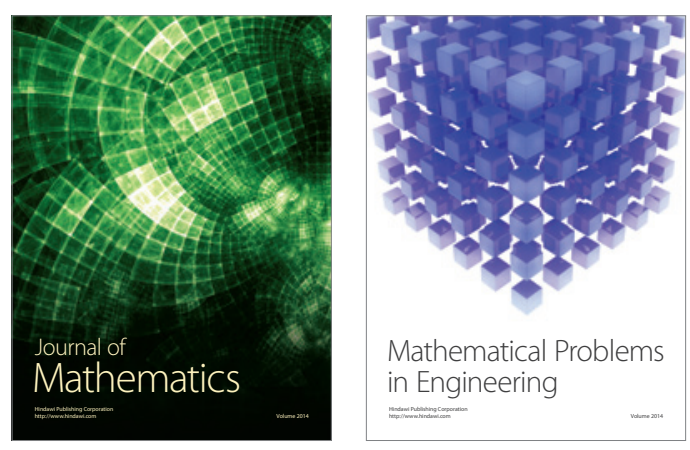

Mathematical Problems in Engineering
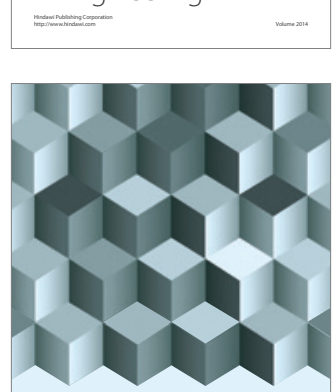

Journal of

Function Spaces
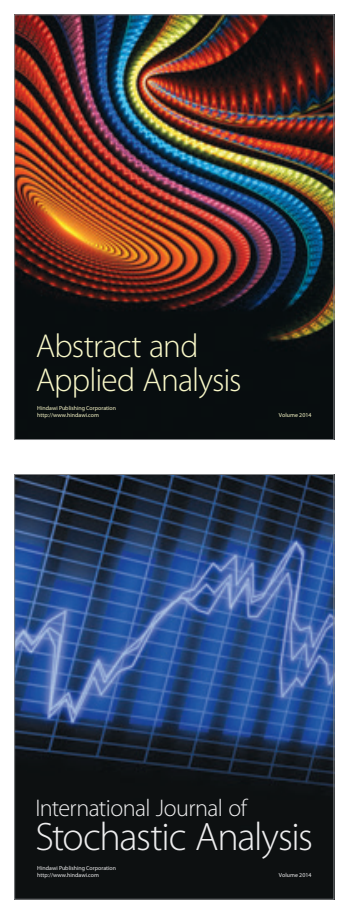

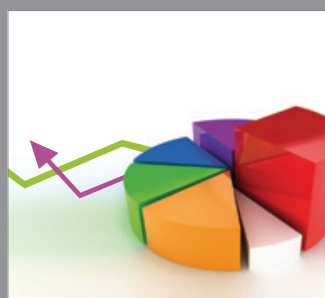

ournal of

Probability and Statistics

Promensencen
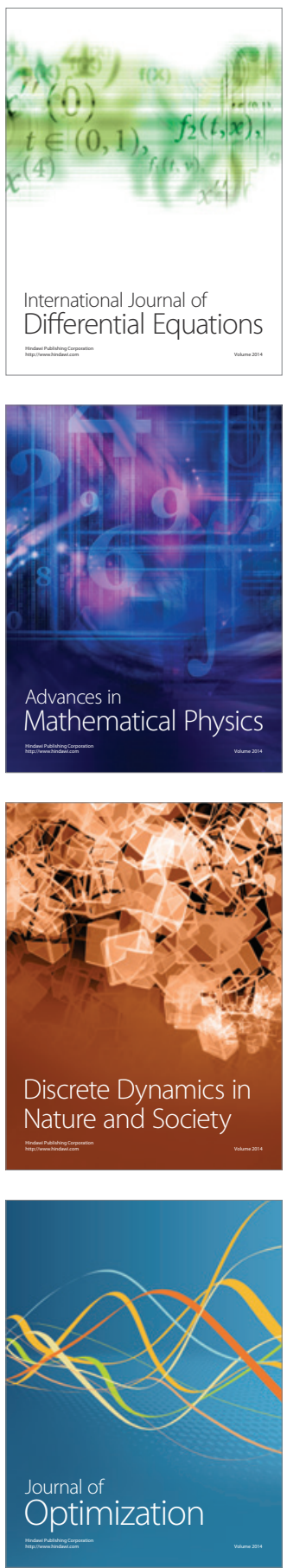